\title{
Diagnostic Performance of Confocal Laser Endomicroscopy for the Detection of Bladder Cancer: Systematic Review and Meta-Analysis
}

\author{
Jie Wu ${ }^{a, b}$ Yu-Chen Wang ${ }^{a, b}$ Wen-Jie Luo ${ }^{a, b}$ Bo Dai ${ }^{a, b}$ Ding-Wei Ye ${ }^{a, b}$ \\ Yi-Ping Zhu ${ }^{\mathrm{a}}$ b \\ ${ }^{a}$ Department of Urology, Fudan University Shanghai Cancer Center, Shanghai, China; \\ ${ }^{b}$ Department of Oncology, Shanghai Medical College, Fudan University, Shanghai, China
}

\section{Keywords}

Confocal laser endomicroscopy · Bladder cancer .

Diagnostic performance $\cdot$ Meta-analysis

\begin{abstract}
Objective: To systematically evaluate the diagnostic efficacy of confocal laser endomicroscopy (CLE) in detection of bladder cancer. Methods: A systematic literature search on CLE in diagnosing bladder cancer in PubMed, Embase, and the Cochrane Library databases was performed. A bivariate meta-regression model was used for meta-analysis to evaluate the pooled diagnostic value of CLE. Results: A total of 5 eligible studies involving 302 lesions were available for this meta-analysis. In a per-lesion analysis, pooled sensitivity, specificity, positive likelihood ratio (PLR), negative likelihood ratio (NLR), diagnostic odds ratio (DOR), and summary receiver-operating curve (SROC) area under the curve (AUC) of CLE for malignant lesions were 0.90 (95\% confidence interval [Cl]: 0.85-0.94), 0.72 (95\% Cl: 0.59-0.82), 3.20 (95\% Cl: $2.14-$ 4.79), 0.14 (95\% Cl: 0.09-0.21), 23.27 (95\% Cl: 11.71-46.25), and 0.91 (95\% Cl: 0.89-0.94), respectively. For low-grade urothelial carcinomas, pooled sensitivity, specificity, PLR, NLR, DOR, and AUC for CLE were 0.72 (95\% Cl: 0.57-0.84), 0.87
\end{abstract}

(95\% Cl: 0.77-0.93), 5.48 (95\% Cl: 3.12-9.62), 0.32 (95\% Cl: $0.20-0.50), 17.19$ (95\% Cl: 8.01-36.89), and 0.85 (95\% Cl: $0.82-0.88)$, respectively. For high-grade urothelial carcinomas, pooled sensitivity, specificity, PLR, NLR, DOR, and AUC for CLE were 0.82 (95\% Cl: 0.62-0.92), 0.84 (95\% Cl: $0.73-$ $0.91), 4.96$ (95\% Cl: $2.58-9.54), 0.22$ (95\% Cl: 0.09-0.52), 22.49 (95\% Cl: 5.33-94.85), and 0.89 (95\% Cl: 0.86-0.91), respectively. Conclusion: CLE is a promising endoscopy technique for real-time tumor grading of bladder cancer.

(c) 2020 S. Karger AG, Basel

\section{Introduction}

Bladder cancer is currently the most prevalent and lethal urinary system malignancy, representing the 11th most commonly diagnosed malignant tumor worldwide [1]. According to the American Cancer Society estimates, about 80,470 new bladder cancer cases were diagnosed and 17,670 bladder cancer deaths occurred in the USA in 2019 [2]. Fortunately, about $75 \%$ of the tumors are con-

Jie-Wu and Yu-Chen Wang contributed equally to this work. karger@karger.com www.karger.com/uin

(c) 2020 S. Karger AG, Basel

Karger ${ }^{\prime}=$
Yi-Ping Zhu

Fudan University Shanghai Cancer Center

Department of Urology

No. 270 Dong'an Road, Shanghai 200032 (China)

fudanzhuyiping@163.com 
fined to the mucosa (pTa, carcinoma in situ [CIS]) or submucosa ( $\mathrm{pT} 1)$, classified as non-muscle-invasive bladder cancer (NMIBC) [3]. However, NMIBC is a disease with a high frequency of early recurrence risk, which can reach $45 \%$ at the first follow-up cystoscopy 3 months after transurethral resection of the bladder tumor (TURBT) [4]. The relatively high rates of prevalence and recurrence make NMIBC a large public health burden. Although current diagnosis of bladder tumor is based on white light cystoscopy (WLC) with histological assessment of resected tissue, WLC has several well-documented shortcomings [5]. In fact, overlooked tumors or inadequate initial resection due to inadequate visualization of WLC significantly affects disease management and patient prognosis.

Hence, in order to improve bladder cancer visualization and detection for NMIBC, several significant advances in endoscopic technique have emerged, including narrow band imaging (NBI), fluorescence cystoscopy, as well as optical coherence tomography (OCT) $[6,7]$. Confocal laser endomicroscopy (CLE) is a unique optical imaging technology. It can provide real-time imaging of cellular architecture and morphology of mucosal lesions with high resolution [8-10]. The advantage of providing histological information during TURBT or cystoscopy to the surgeon makes CLE a more promising technique for conservative bladder cancer management. After first being introduced in 2004, the application of CLE has expanded from gastrointestinal and biliopancreatic diseases to pulmonology and urology [11-13].

Recently, CLE has been reported to have a great diagnostic accuracy for bladder cancer in several studies [9, 14-16]. However, a comprehensive overview of the diagnostic performance of CLE of bladder cancer has not been reported. Therefore, we conducted this review to systematically evaluate the diagnostic value of CLE in diagnosis of bladder cancer.

\section{Materials and Methods}

\section{Search Strategies}

This meta-analysis was conducted according to the Preferred Reporting Items for Systematic Reviews and Meta-Analyses statement [17]. An online search of PubMed, Embase, and the Cochrane Library databases for relevant works in the English language was conducted using the following search terms: ("confocal laser endomicroscopy" OR "fibered confocal microscopy" OR "confocal endoscopy" OR "CLE") AND ("bladder cancer" OR "urinary bladder tumor" OR "bladder carcinoma"). The cutoff date for the database inclusion was January 12, 2020. References from identified studies were examined for additional eligible papers.

\section{Study Selection Criteria}

Two independent reviewers (J.W. and Y.-C.W.) conducted the literature search and study selection. Disagreement was resolved by a third reviewer (Y.-P.Z.). Studies were included for initial evaluation according to the following criteria: (1) the study was either a clinical trial or an observational study (case-control or cohort); (2) sensitivity and specificity were provided or contained sufficient data to construct a $2 \times 2$ contingency table, including true positive, false positive, false negative, and true negative; and (3) diagnosis of bladder cancer using histopathology as a reference standard. Reviews, case reports, letters, or conference abstracts were excluded.

\section{Data Extraction and Quality Assessment}

Two independent investigators extracted the following variables from each eligible study: the name of the first author, year of publication, number of enrolled patients, number of included lesions, mean age of patients, sex ratio, number of endoscopists and their experience, and diagnostic procedure. The quality of the included studies and the risk of bias were evaluated using the Quality Assessment of Diagnostic Accuracy Studies-2 (QUADAS-2) tool by 2 independent reviewers [18].

\section{Statistical Analysis}

STATA version 15.0 (StataCorp2, College Station, TX, USA) was used to conduct all statistical calculations. Pooled sensitivity, specificity, positive likelihood ratio (PLR), negative likelihood ratio (NLR), and diagnostic odds ratio (DOR) with corresponding 95\% confidence intervals (CIs) were calculated to evaluate the diagnostic value of CLE in diagnosing bladder cancer. Pooled results were estimated using a bivariate meta-regression model [19]. Heterogeneity across studies was estimated using the Cochrane $Q$ and inconsistency $\left(I^{2}\right)$ tests. Statistically significant heterogeneity among studies was assumed for $Q$ test $p$ values $<0.10$ and $I^{2}>50 \%$. The symmetric summary receiver-operating curve (SROC) was used to summarize the overall accuracy of CLE in distinguishing normal tissues from malignant lesions and diagnosing low- and high-grade urothelial carcinomas. Diagnostic effect was determined using area under the curve (AUC) calculated from the SROC curve, where values $\geq 0.8$ demonstrated a good diagnostic performance. A funnel plot was constructed to evaluate the potential publication bias in selected studies using Deeks' asymmetry test.

\section{Results}

\section{Study Characteristic and Quality Assessment}

Figure 1 shows the literature search flow for the report. Briefly, 69 potentially relevant studies were identified in the initial search and 64 studies were excluded for a variety of reasons. A total of 5 eligible studies [9, 14-16, 20], including 302 lesions, met the inclusion criteria. The characteristics of the eligible studies are presented in Table 1. Quality assessment of each study based on the QUADAS-2 criteria is presented in Table 2. The overall quality of the studies was satisfactory. 
Fig. 1. Flow diagram of identifying eligible

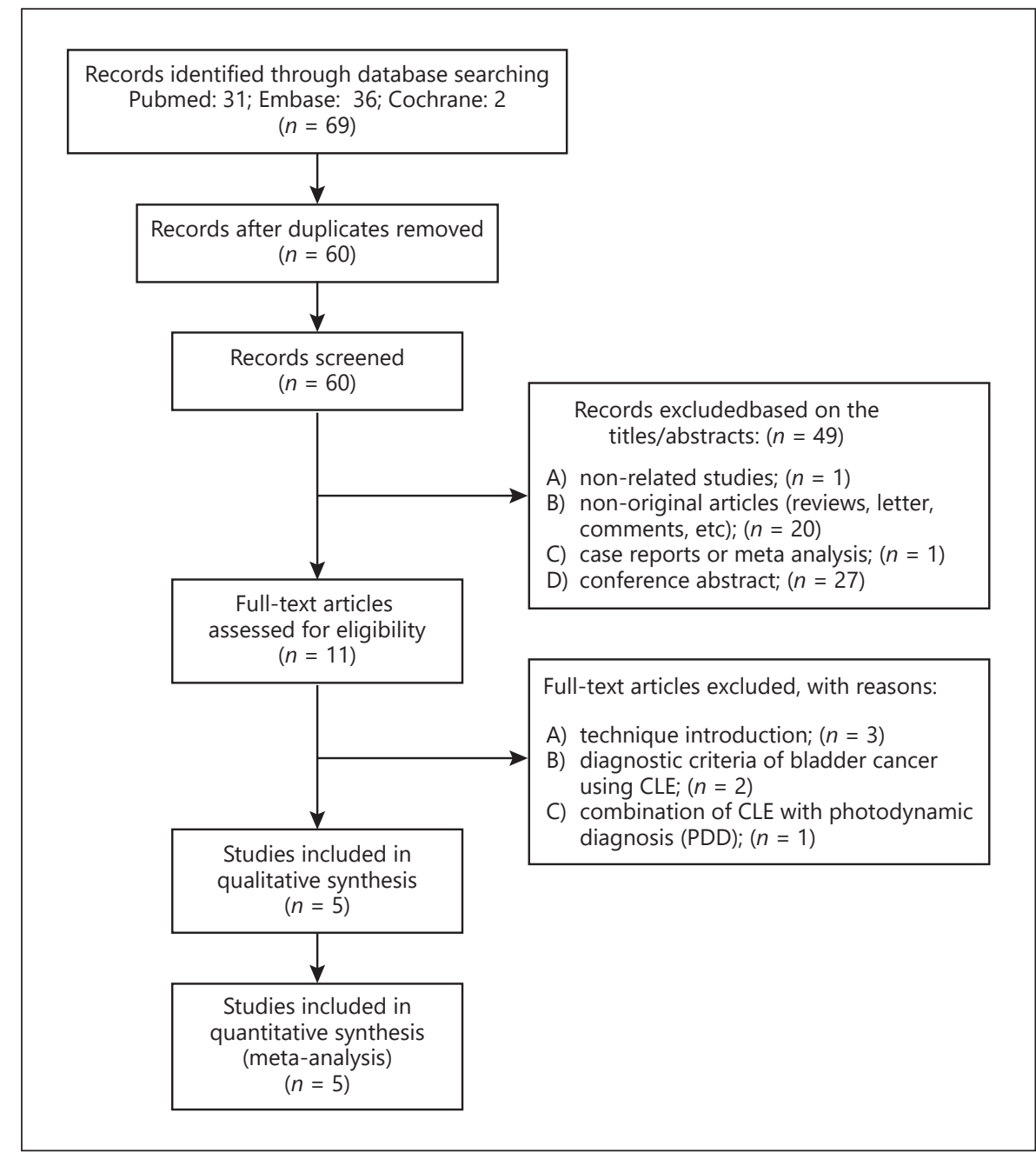
studies and exclusion criteria.

Table 1. Basic characteristics of the included studies

\begin{tabular}{|c|c|c|c|c|c|c|c|c|c|}
\hline Study & Year & $\begin{array}{l}\text { Patients, } \\
n\end{array}$ & $\begin{array}{l}\text { Lesions, } \\
n\end{array}$ & $\begin{array}{l}\text { Mean } \\
\text { age }\end{array}$ & $\begin{array}{l}\text { Male/ } \\
\text { female }\end{array}$ & Endoscopist & $\begin{array}{l}\text { Endoscopist, } \\
n\end{array}$ & Diagnostic procedure & Ref \\
\hline Lucas & 2019 & 53 & 66 & $\mathrm{n} / \mathrm{a}$ & $\mathrm{n} / \mathrm{a}$ & Trained endoscopist & 2 & Blinded; CLE & {$[20]$} \\
\hline Lee & 2019 & 75 & 119 & 68 & $90 / 29$ & Trained endoscopist & 1 & Blinded; CLE & {$[15]$} \\
\hline Liem & 2020 & 53 & 65 & 70 & $39 / 14$ & Trained endoscopist & 3 & Blinded; WLC + CLE & {$[14]$} \\
\hline Chang & 2013 & $\mathrm{n} / \mathrm{a}$ & 31 & $\mathrm{n} / \mathrm{a}$ & $\mathrm{n} / \mathrm{a}$ & Experienced endoscopist & 2 & Blinded; WLC + CLE & [9] \\
\hline
\end{tabular}

n/a, not available; trained endoscopist, endoscopist who trained with a CLE training program; experienced endoscopist, endoscopist who involved in the protocol development of CLE for bladder cancer. CLE, confocal laser endomicroscopy; WLC, white light cystoscopy.

Diagnostic Value of CLE for NMIBC

Diagnostic Value for Malignant Lesions

Data from the 5 studies with 302 lesions were analyzed. The pooled sensitivity and specificity of CLE for malignant lesions were 0.90 (95\% CI: 0.85-0.94, $I^{2}=$ $26.88 \%$ ) and 0.72 (95\% CI: $0.59-0.82, I^{2}=36.81 \%$ ), respectively (Fig. 2a, b). The pooled PLR was 3.20 (95\% CI: $\left.2.14-4.79, I^{2}=0.0 \%\right)$, and pooled NLR was 0.14 


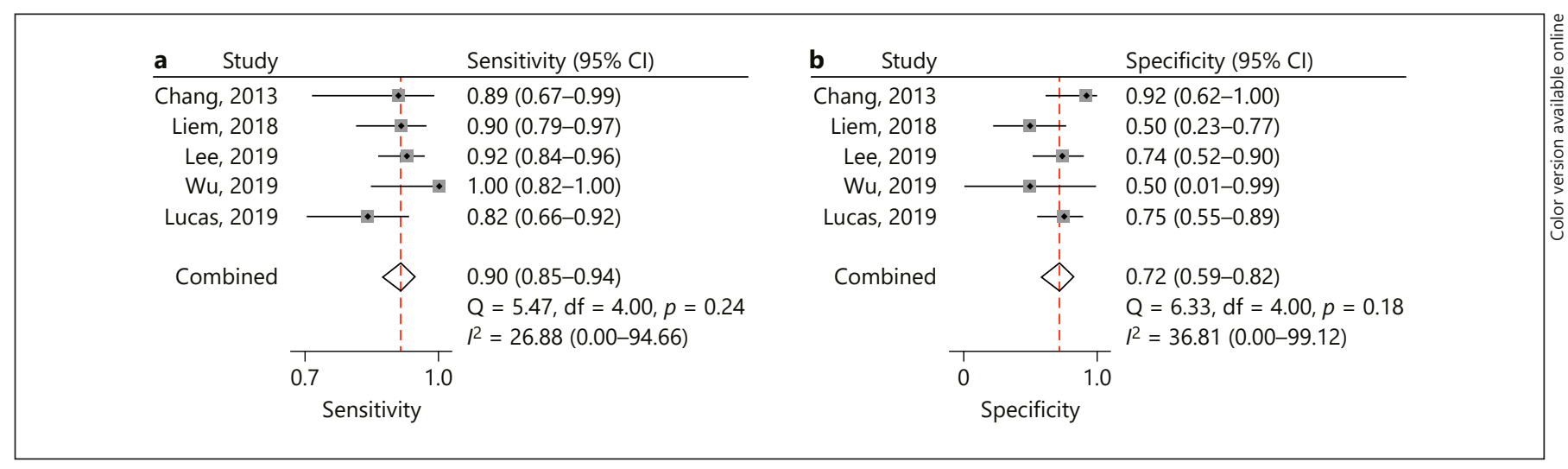

Fig. 2. Meta-analysis of diagnostic performance of CLE for malignant lesions. a Forest plots of the sensitivity. b Forest plots of the specificity.

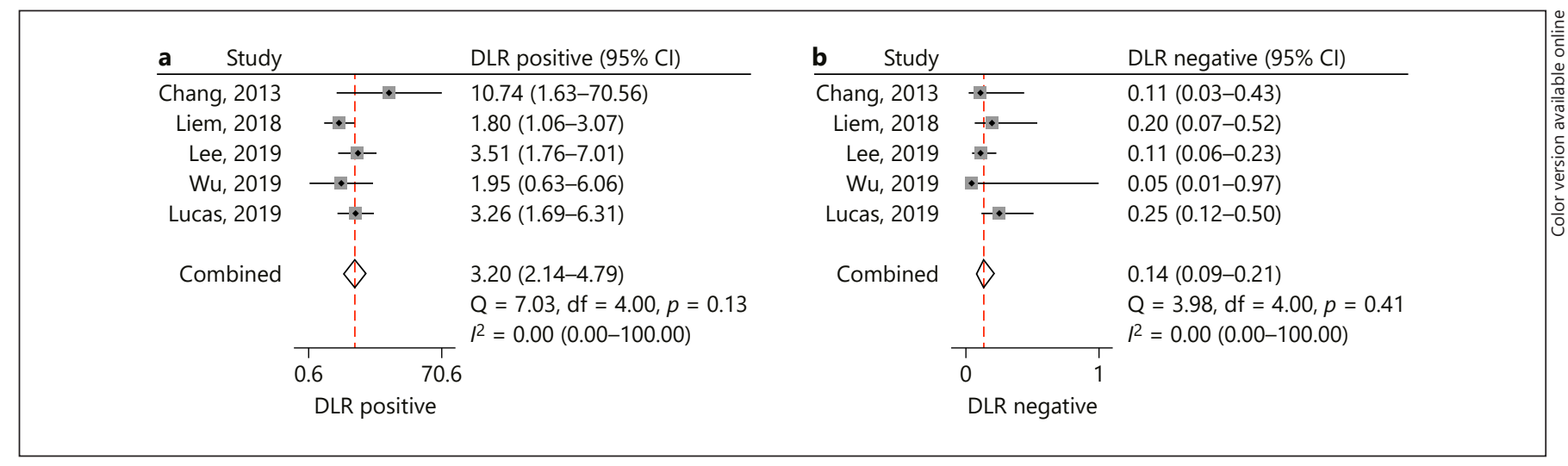

Fig. 3. Meta-analysis of diagnostic performance of CLE for malignant lesions. a Forest plots of PLR. b Forest plots of NLR.

Table 2. Quality of articles using the QUADAS-2 tool

\begin{tabular}{|c|c|c|c|c|c|c|c|c|}
\hline \multirow[t]{2}{*}{ Study } & \multirow[t]{2}{*}{ Year } & \multicolumn{4}{|c|}{ Risk of bias } & \multicolumn{3}{|c|}{ Applicability concerns } \\
\hline & & $\begin{array}{l}\text { patient } \\
\text { selection }\end{array}$ & index test & $\begin{array}{l}\text { reference } \\
\text { standard }\end{array}$ & $\begin{array}{l}\text { flow and } \\
\text { timing }\end{array}$ & $\begin{array}{l}\text { patient } \\
\text { selection }\end{array}$ & index test & $\begin{array}{l}\text { reference } \\
\text { standard }\end{array}$ \\
\hline Lucas & 2019 & Low risk & Low risk & Low risk & Low risk & Low risk & Low risk & Low risk \\
\hline $\mathrm{Wu}$ & 2019 & Low risk & Low risk & Low risk & Low risk & Low risk & Low risk & Low risk \\
\hline Lee & 2019 & Low risk & Low risk & Low risk & Low risk & Low risk & Low risk & Low risk \\
\hline Liem & 2020 & Low risk & Low risk & Low risk & Low risk & Low risk & Low risk & Low risk \\
\hline Chang & 2013 & High risk & Low risk & Low risk & Low risk & Low risk & Low risk & Low risk \\
\hline
\end{tabular}

QUADAS-2, Quality Assessment of Diagnostic Accuracy Studies-2.

(95\% CI: $0.09-0.21, I^{2}=0.0 \%$ ) (Fig. 3a, b). The pooled DOR and AUC were 23.27 (95\% CI: 11.71-46.25, $I^{2}=$ 70.91\%) and 0.91 (95\% CI: 0.89-0.94), respectively (Fig. 4, 5).
Diagnostic Value for Low-Grade Urothelial Carcinomas

Pooled sensitivity, specificity, PLR, NLR, and DOR for CLE in diagnosing low-grade urothelial carcinomas are 


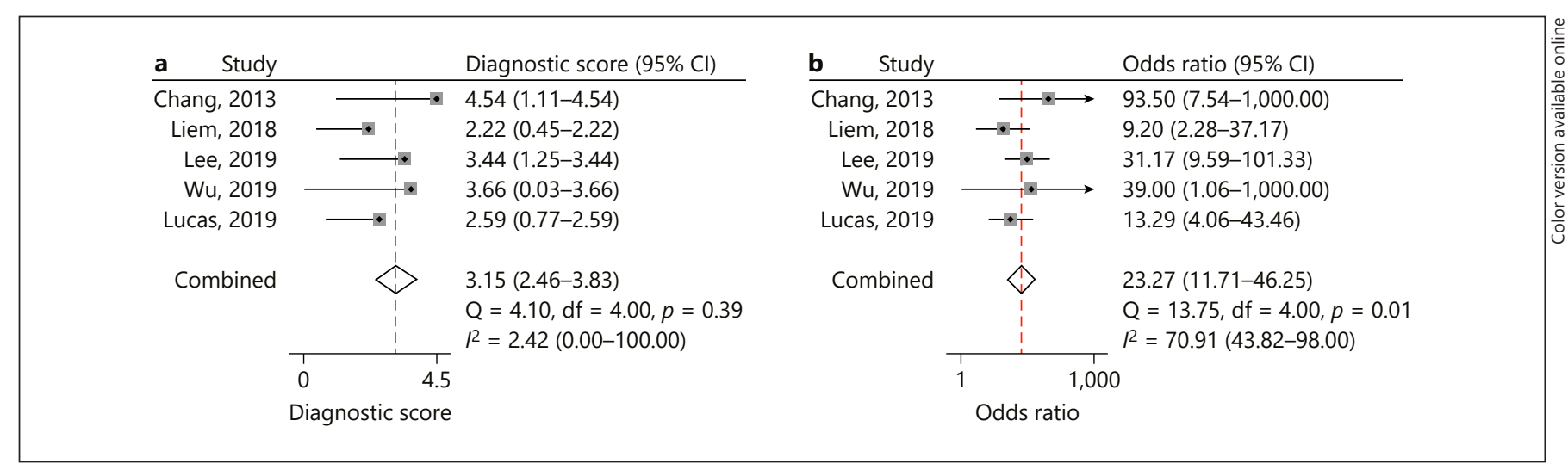

Fig. 4. Meta-analysis of diagnostic performance of CLE for malignant lesions. a Forest plots of diagnostic score. b Forest plots of DOR.

presented in Fig. 6. The pooled sensitivity was 0.72 (95\% CI: $\left.0.57-0.84, I^{2}=0.0 \%\right)$ and pooled specificity was 0.87 (95\% CI: $0.77-0.93, I^{2}=62.76 \%$ ) (Fig. 6a). The pooled PLR was 5.48 (95\% CI: 3.12-9.62, $\left.I^{2}=0.0 \%\right)$ and pooled NLR was 0.32 (95\% CI: $0.20-0.50, I^{2}=0.0 \%$ ) (Fig. 6b). The pooled DOR was $17.19\left(95 \% \mathrm{CI}: 8.01-36.89, I^{2}=46.92 \%\right)$ and AUC was 0.85 (95\% CI: 0.82-0.88) (Fig. 6c, d).

Diagnostic Value for High-Grade Urothelial

\section{Carcinomas}

CLE had a pooled sensitivity of 0.82 (95\% CI: $0.62-$ $\left.0.92, I^{2}=72.82 \%\right)$ and pooled specificity of 0.84 (95\% CI: $\left.0.73-0.91, I^{2}=46.78 \%\right)$ for diagnosing high-grade urothelial carcinomas (Fig. 7a). The pooled PLR was 4.96 (95\% CI: $\left.2.58-9.54, I^{2}=26.98 \%\right)$ and pooled NLR was 0.22 (95\% CI: 0.09-0.52, $I^{2}=75.30 \%$ ) (Fig. 7b). The pooled DOR was 22.49 (95\% CI: 5.33-94.85, $\left.I^{2}=99.83 \%\right)$ and AUC was 0.89 (95\% CI: 0.86-0.91) (Fig. 7c, d).

\section{Publication Bias}

The funnel plots based on Deeks' asymmetry test were not significantly asymmetrical, indicating that there did not exist significant publication bias (Fig. 8).

\section{Discussion}

Currently, WLC is a crucial technique in bladder cancer management and histopathology is still considered to be the gold standard method in bladder cancer diagnosis [1]. However, WLC cannot provide histopathological information and cannot detect fat lesions effectively, especially discriminating between inflammation and malig-



Fig. 5. SROC curve of CLE for malignant lesions. SROC, summary receiver-operating curve; CLE, confocal laser endomicroscopy; AUC, area under the curve.

nant lesions [5]. In addition, biopsy requires complicated and time-consuming procedures and is, thus, not readily available to provide histopathological information during cystoscopy. Thus, it is important to develop novel endoscopic techniques to improve the detection and disease management of bladder cancer. CLE is a high-resolution optical imaging technology known as "optical biopsy," owing to its ability to generate a 500-1,000 magnification 

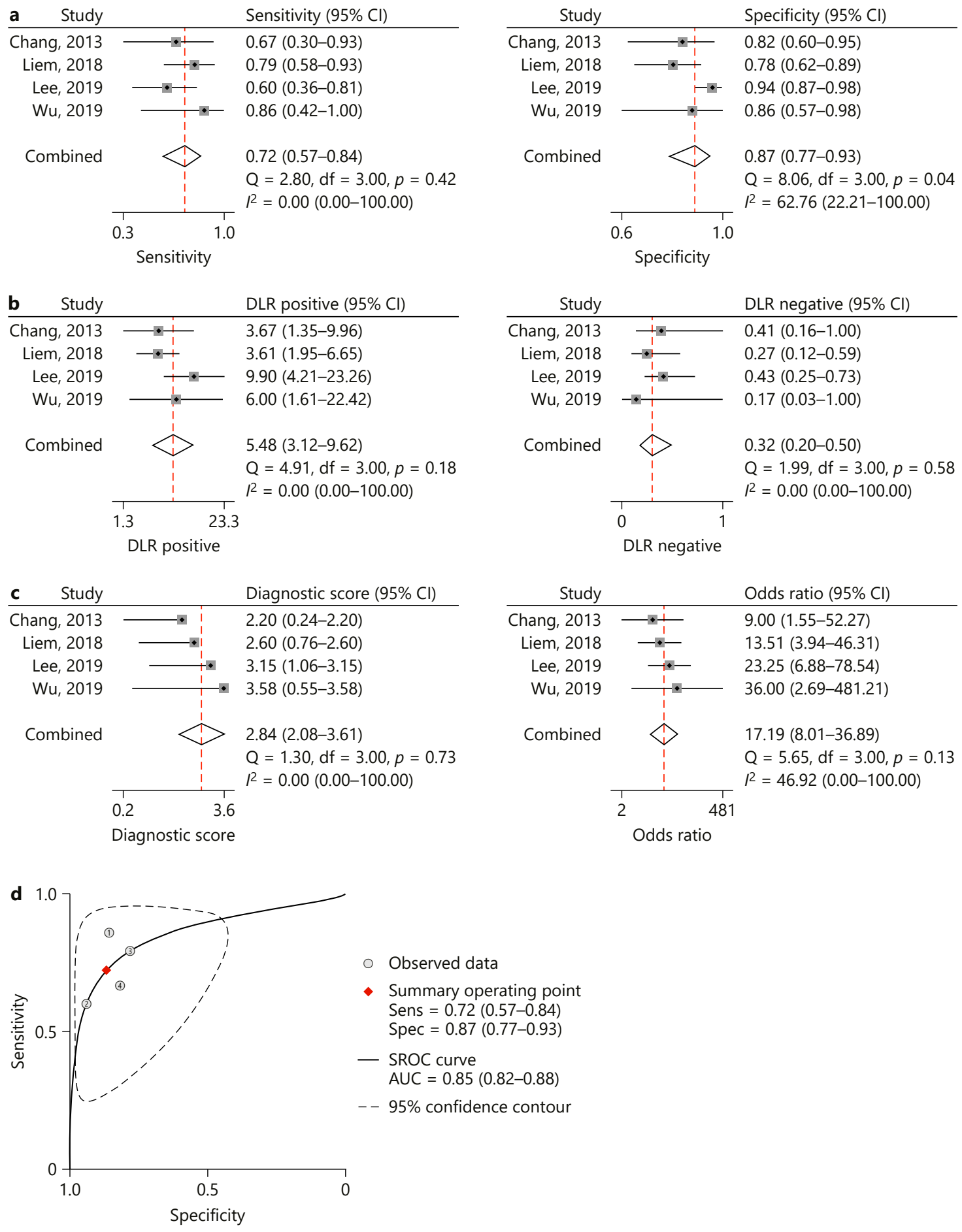

Fig. 6. Meta-analysis of diagnostic performance of CLE for low-grade urothelial carcinomas. Forest plots of sensitivity and specificity (a), forest plots of PLR and NLR (b), forest plots of diagnostic score and DOR (c), and SROC curve of CLE for low-grade urothelial carcinomas (d). CLE, confocal laser endomicroscopy; PLR, positive likelihood ratio; NLR, negative likelihood ratio; DOR, diagnostic odds ratio; SROC, summary receiver-operating curve; AUC, area under the curve. 

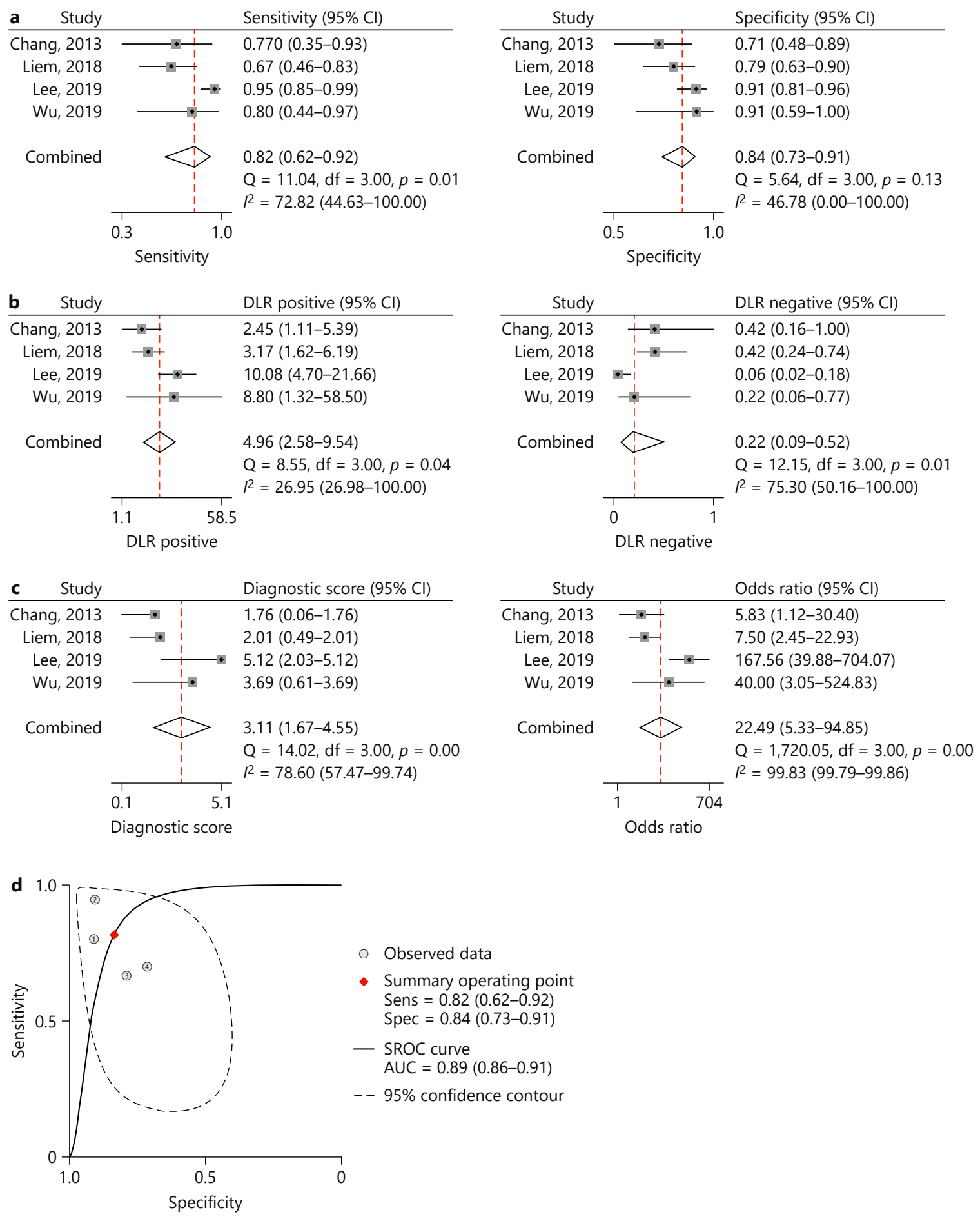

Fig. 7. Meta-analysis of diagnostic performance of CLE for high-grade urothelial carcinomas. Forest plots of sensitivity and specificity (a), forest plots of PLR and NLR (b), forest plots of diagnostic score and DOR (c), and SROC curve of CLE for high-grade urothelial carcinomas (d). CLE, confocal laser endomicroscopy; PLR, positive likelihood ratio; NLR, negative likelihood ratio; DOR, diagnostic odds ratio; SROC, summary receiver-operating curve; AUC, area under the curve. 




Fig. 8. Deeks' funnel plot to evaluate the publication bias of selected studies of malignant lesions (a), low-grade urothelial carcinomas (b), and high-grade urothelial carcinomas (c).

of high-accuracy dynamic histological tissue imaging [21]. CLE was the first and now is widely used in gastrointestinal endoscopy and has been demonstrated to have a high diagnostic value [22]. After Sonn et al. [8] first characterized the CLE features of bladder cancer in 2009, the application of CLE in tract urothelial carcinoma diagnosis has been evaluated and the diagnostic criteria for bladder urothelium based on CLE have been initially established $[9,23,24]$.

To our knowledge, this is the first meta-analysis to summarize the available evidence for the diagnostic value of CLE in bladder cancer characterization. As mentioned before, summary sensitivity and specificity of CLE for malignant lesions were 0.90 and 0.72 , respectively. When compared to other optical imaging techniques, CLE had a similar sensitivity but a lower specificity. NBI is an optical image enhancement technique that can differentially penetrate mucosa to enhance the visibility of mucosal capillaries and delicate tissue surface structures. A metaanalysis published in 2012 showed that the pooled sensitivity and specificity of NBI diagnosis of bladder cancer were 0.94 (95\% CI: 0.91-0.96) and 0.85 (95\% CI: 0.810.88 ), respectively, in a per-person analysis [25]. OCT is a biomedical optical imaging technique that can provide real-time and cross-sectional images of tissue morphology. In a meta-analysis published in 2018, an excellent diagnostic accuracy of OCT in detection of bladder cancer was shown, where the pooled sensitivity and specificity were 0.96 (95\% CI: $0.94-0.98)$ and 0.82 (95\% CI: $0.80-$
$0.85)$, respectively, on a per-patient level [26]. In the present study, the specificity of CLE was a little disappointing. Observer subjectivity and deficient diagnostic criteria may be the main reasons. Nevertheless, the way to assess a novel diagnostic technique is not only via sensitivity or specificity but also using the likelihood ratio and SROC. The pooled DOR and AUC of CLE-based diagnosis were 23.27 and 0.91 , respectively. The pooled PLR was 3.20 and pooled NLR was 0.14 . These statistical results indicated that CLE still had a high diagnostic precision in bladder cancer.

Moreover, the ability to distinguish the structure of microstructures or individual cells makes CLE-based real-time tumor grading of urothelial carcinoma possible, presenting the greatest advantage of CLE. This advantage could not only confirm the radicality of TURBT, thus reducing the recurrence rate, but also facilitate the followup quality in NMIBC patients. As mentioned above, CLE pooled sensitivity and specificity for low-grade urothelial carcinomas were 0.72 and 0.87 , respectively. CLE pooled sensitivity and specificity for high-grade urothelial carcinomas were 0.82 and 0.84 , respectively. The diagnostic precision of CLE for bladder carcinoma grading is inferior to that for malignant lesions. However, since systemic CLE diagnostic criteria for bladder lesions have not been established, the diagnostic performance of CLE for tumor grading of urothelial carcinoma is quite satisfactory. CLE-based bladder cancer diagnosis relies on microarchitectural and cellular features. Nevertheless, 
translation of images into diagnosis is not straightforward. However, it was believed that after the accomplishment of multicenter collaborations for larger clinical trials to refine the proposed CLE criteria and develop a diagnostic nomogram, the diagnostic performance of CLE in tumor grading of urothelial carcinoma would be improved and CLE could be routinely used for NMIBC diagnosis.

In the current study, CLE was shown to be a reliable method for the identification of malignant bladder lesions. However, it also had several limitations. First, the diagnostic precision of CLE was a little lower than that of other novel endoscopic imaging technology or the CLE applied in other organs [22]. The first reason might have been due to the lack of diagnostic criteria for CLE in bladder cancer. Chang et al. [9] proposed CLE-based diagnostic criteria for bladder lesions in 2013, where architectural and cellular features were shown to be able to effectively identify benign and abnormal lesions. Liem et al. [14] then validated and improved these criteria in 2018, suggesting that organization of cells, cellular morphology, and definition of cell borders were the differential features for tumor grading of bladder cancer. The diagnosis accuracy of CLE remained unsatisfactory, indicating that the established CLE criteria needed to be refined. Another reason may lie in the experience of the observers. As a novel technique targeting optical biopsy, images obtained in CLE are much more complicated and unfamiliar to most urologists. Systematic training and substantial practice are needed, since online training provided by the company is obviously not enough [9]. Third, CIS, a highgrade malignant lesion confined to the mucosa, contributes to greater mortality and morbidity due to its high potential to recur and progress. In that case, accurate diagnosis of CIS is particularly important but still remains challenging [27]. However, there are few studies enrolling an adequate number of CIS patients to evaluate the diagnostic value of CLE in detection of CIS. There was only one study that evaluated 21 CIS biopsy sites and found that CIS detection rate using CLE was $83.3 \%$, suggesting that CLE may be a solution for differential diagnosis between CIS and inflammatory tissue. More multicenter randomized studies are required to confirm these conclusions [5]. In addition, CLE may not be cost-effective compared to WLC, which may limit large-scale application of CLE.

Combination of CLE with other endoscopic techniques could be a better choice and may greatly alter current bladder cancer management. Marien et al. [28] evaluated the feasibility of using CLE with 2 fluorophores (fluorescein and hexyl aminolevulinate) for bladder cancer diagnosis and found that sensitivity and specificity of this combination can reach 80 and $100 \%$, respectively. However, application of CLE combined with other endoscopic techniques is still in the early stages, and the advantage of various combinations remains to be further investigated.

Each meta-analysis has some unavoidable limitations. The main limitation of the current meta-analysis is that it relied on only 5 studies with limited samples. Therefore, it might lack the power to illustrate the diagnostic value of CLE in bladder cancer. Second, in virtue of different inclusion and exclusion criteria as well as observer experience bias, there was a statistical heterogeneity in some pooled analyses. Third, since most data are available on a lesion level, it was not possible to estimate the diagnostic value of CLE on a per-patient basis, although data on a patient level may be more relevant clinically. Finally, nonEnglish language articles were excluded, which may lead to a language bias.

In summary, existing evidence demonstrates that CLE is an effective technique to diagnose bladder cancer with high precision. CLE could be considered a promising endoscopy technique for real-time tumor grading of NMIBC and a complement to improve the diagnostic accuracy of WLC. Application of CLE in bladder cancer is still in the early stages, and further improvement and validation from prospective multicenter studies with a larger cohort for CLE diagnosis criteria are expected.

\section{Acknowledgement}

This work was supported by the National Natural Science Foundation of China (Project 81772706).

\section{Disclosure Statement}

The authors declare no conflict of interest.

\section{Author Contributions}

J.-W. conducted the data collection and quality assessment and was a major contributor in manuscript writing. Y.-C.W. performed the statistical analyses. W.-J.L. and B.-D. were involved in the manuscript editing. Y.-P.Z. and D.-W.Y. conceived and designed the study. All authors read and approved the final manuscript. 


\section{References}

1 Babjuk M, Burger M, Comperat EM, Gontero P, Mostafid AH, Palou J, et al. European association of urology guidelines on non-muscle-invasive bladder cancer (TaT1 and carcinoma in situ)-2019 update. Eur Urol. 2019 Nov;76(5):639-657.

2 Siegel RL, Fedewa SA, Miller KD, GodingSauer A, Pinheiro PS, Martinez-Tyson D, et al. Cancer statistics for Hispanics/Latinos, 2015. CA Cancer J Clin. 2015;65(6):457-80.

3 Burger M, Catto JW, Dalbagni G, Grossman HB, Herr H, Karakiewicz P, et al. Epidemiology and risk factors of urothelial bladder cancer. Eur Urol. 2013 Feb;63(2):234-41.

4 Brausi M, Collette L, Kurth K, van der Meijden AP, Oosterlinck W, Witjes JA, et al. Variability in the recurrence rate at first follow-up cystoscopy after TUR in stage Ta T1 transitional cell carcinoma of the bladder: a combined analysis of seven EORTC studies. Eur Urol. 2002 May;41(5):523-31.

5 Lee CS, Yoon CY, Witjes JA. The past, present and future of cystoscopy: the fusion of cystoscopy and novel imaging technology. BJU Int. 2008 Nov; 102 (9 Pt B):1228-33.

6 Raharja PAR, Hamid ARAH, Mochtar CA, Umbas R. Recent advances in optical imaging technologies for the detection of bladder cancer. Photodiagnosis Photodyn Ther. 2018 Dec;24:192-7.

7 Tully K, Palisaar RJ, Brock M, Bach P, von Landenberg N, Löppenberg B, et al. Transurethral resection of bladder tumours: established and new methods of tumour visualisation. Transl Androl Urol. 2019 Feb;8(1):25-33.

8 Sonn GA, Jones SN, Tarin TV, Du CB, Mach $\mathrm{KE}$, Jensen KC, et al. Optical biopsy of human bladder neoplasia with in vivo confocal laser endomicroscopy. J Urol. 2009 Oct;182(4): 1299-305.

9 Chang TC, Liu JJ, Hsiao ST, Pan Y, Mach KE, Leppert JT, et al. Interobserver agreement of confocal laser endomicroscopy for bladder cancer. J Endourol. 2013 May;27(5):598-603.

10 Chen SP, Liao JC. Confocal laser endomicroscopy of bladder and upper tract urothelial carcinoma: a new era of optical diagnosis? Curr Urol Rep. 2014 Sep;15(9):437.
11 Karia K, Kahaleh M. A review of probe-based confocal laser endomicroscopy for pancreaticobiliary disease. Clin Endosc. 2016 Sep; 49(5):462-6.

12 Wijmans L, d'Hooghe JN, Bonta PI, Annema JT. Optical coherence tomography and confocal laser endomicroscopy in pulmonary diseases. Curr Opin Pulm Med. 2017 May; 23(3):275-83.

13 Xiong YQ, Ma SJ, Hu HY, Ge J, Zhou LZ, Huo ST, et al. Comparison of narrow-band imaging and confocal laser endomicroscopy for the detection of neoplasia in Barrett's esophagus: a meta-analysis. Clin Res Hepatol Gastroenterol. 2018 Feb;42(1):31-9.

14 Liem E, Freund JE, Savci-Heijink CD, de la Rosette J, Kamphuis GM, Baard J, et al. Validation of confocal laser endomicroscopy features of bladder cancer: the next step towards real-time histologic grading. Eur Urol focus. 2020;6(1):81-7.

15 Lee J, Jeh SU, Koh DH, Chung DY, Kim MS, Goh HJ, et al. Probe-based confocal laser endomicroscopy during transurethral resection of bladder tumors improves the diagnostic accuracy and therapeutic efficacy. Ann Surg Oncol. 2019 Apr;26(4):1158-65.

16 Wu J, Wang YC, Dai B, Ye DW, Zhu YP. Optical biopsy of bladder cancer using confocal laser endomicroscopy. Int Urol Nephrol. 2019;51(9):1473-79.

17 Moher D, Liberati A, Tetzlaff J, Altman DG, Group P. Preferred reporting items for systematic reviews and meta-analyses: the PRISMA statement. PLoS Med. 2009 Jul 21; 6(7):e1000097.

18 Whiting PF, Rutjes AW, Westwood ME, Mallett S, Deeks JJ, Reitsma JB, et al. QUADAS-2: a revised tool for the quality assessment of diagnostic accuracy studies. Ann Intern Med. 2011 Oct 18;155(8):529-36.

19 Reitsma JB, Glas AS, Rutjes AW, Scholten RJ, Bossuyt PM, Zwinderman AH. Bivariate analysis of sensitivity and specificity produces informative summary measures in diagnostic reviews. J Clin Epidemiol. 2005 Oct;58(10): 982-90.
20 Lucas M, Liem EIML, Savci-Heijink CD, Freund JE, Marquering HA, van Leeuwen TG, et al. Toward automated in vivo bladder tumor stratification using confocal laser endomicroscopy. J Endourol. 2019 Nov;33(11): 930-7.

21 Wiesner C, Jäger W, Salzer A, Biesterfeld S, Kiesslich R, Hampel C, et al. Confocal laser endomicroscopy for the diagnosis of urothelial bladder neoplasia: a technology of the future? BJU Int. 2011 Feb;107(3):399-403.

22 Dong YY, Li YQ, Yu YB, Liu J, Li M, Luan XR. Meta-analysis of confocal laser endomicroscopy for the detection of colorectal neoplasia. Colorectal Dis. 2013 Sep;15(9):e488-95.

23 Wu K, Liu JJ, Adams W, Sonn GA, Mach KE, Pan Y, et al. Dynamic real-time microscopy of the urinary tract using confocal laser endomicroscopy. Urology. 2011 Jul;78(1):225-31.

24 Breda A, Territo A, Guttilla A, Sanguedolce F, Manfredi M, Quaresima L, et al. Correlation between confocal laser endomicroscopy (cellvizio $^{\varpi}$ ) and histological grading of upper tract urothelial carcinoma: a step forward for a better selection of patients suitable for conservative management. European urology focus. 2018 Dec;4(6):954-9.

25 Zheng C, Lv Y, Zhong Q, Wang R, Jiang Q. Narrow band imaging diagnosis of bladder cancer: systematic review and meta-analysis. BJU Int. 2012 Dec;110(11 Pt B):E680-7.

26 Huang J, Ma X, Zhang L, Jia H, Wang F. Diagnostic accuracy of optical coherence tomography in bladder cancer patients: a systematic review and meta-analysis. Mol Clin Oncol. 2018 Apr;8(4):609-12.

27 Subiela JD, Rodriguez Faba O, Guerrero Ramos F, Vila Reyes H, Pisano F, Breda A, et al. Carcinoma in situ of the urinary bladder: a systematic review of current knowledge regarding detection, treatment, and outcomes. Eur Urol Focus. 2019 Mar 22.

28 Marien A, Rock A, Maadarani KE, Francois C, Gosset P, Mauroy B, et al. Urothelial tumors and dual-band imaging: a new concept in confocal laser endomicroscopy. J Endourol. 2017 May;31(5):538-44. 